\title{
LUDWIG FEUERBACH: L’UOMO E LA SUA ALIMENTAZIONE
}

Francesco Tomasoni ${ }^{1}$

Nel 2015 si è svolto a Milano l'Expo, una manifestazione fieristica dal titolo "Nutrire il pianeta. Energia per la vita" che in 184 giorni e con 145 nazioni partecipanti, fra cui ovviamente il Brasile, ha avuto 21 milioni di visitatori. Sull'onda di questo evento è tornato di attualità il celebre motto di Feuerbach: «L'uomo è (ist) ciò che mangia (isst)». Nella lingua tedesca l'assonanza fra le terze persone del verbo essere e del verbo mangiare è evidente e suggerisce una stretta relazione fra essere e mangiare. Non a caso i critici dell'epoca vi videro un'espressione di rozzo materialismo, che poteva essere avvicinata alla frase di Karl Vogt: «I pensieri stanno press'a poco nel medesimo rapporto col cervello, come la bile al fegato o l'urina alle reni» ${ }^{2}$. Vogt era ben consapevole di usare un'espressione «in un certo senso rozza», ma intendeva dire che «tutte le attività psichiche» erano «solo funzioni della sostanza cerebrale» ${ }^{3}$.

Eppure l'assonanza fra essere e mangiare era già stata messa in rilievo più di sessant'anni prima da un teologo protestante di tendenze razionalistiche, Friedrich Gedike, in un articolo della prestigiosa rivista "Berlinische Monatsschrift", in cui comparvero anche gli interventi di Kant, Mendelssohn e altri sul significato dell'illuminismo. Già nel titolo ${ }^{4}$ egli evidenziava l'assonanza e mostrava che, ancor più che nel tedesco, in latino e in greco i due verbi coincidevano per alcune forme della flessione. Non si trattava dunque di un caso, bensì dell'effetto di un processo naturale. Per i popoli primitivi i concetti astratti erano troppo ardui, perciò al loro posto subentravano riferimenti materiali. Così «per l'uomo rozzo e ancora del tutto sensuale il concetto di essere, nei tempi della prima origine delle lingue, era troppo sottile e remoto [...] al contrario il concetto del mangiare era certamente uno dei primi sviluppatisi in lui» ${ }^{5}$. Poteva dunque argomentare nel modo seguente: mangia, dunque è (in latino: est, ergo est). La terza persona, nella quale la coincidenza era perfetta, rifletteva la mentalità primitiva secondo cui il soggetto osservava le cose fuori di sé, ma non era ancora giunto all'autocoscienza. L'identità fra essere e mangiare aveva anche indotto i primitivi a concludere che se gli dei esistevano, dovevano mangiare. Da qui i banchetti sacrificali, in cui si invitavano le divinità a condividere la mensa con gli uomini. Quanto più però questi si convincevano della superiorità degli dei, tanto più offrivano loro cibi preziosi,

Università del Piemonte Orientale, Vercelli.

Karl Vogt, Physiologische Briefe für Gebildete aller Stände, Giessen, Ricker'sche Buchhandlung, 1854², p. 323.

Ibid.

Friedrich Gedike, Über ißt und ist. Ein Beitrag zur Erklärung der Opfer, „Berlinische Monatsschrift“, Hrsg. F. Gedike u. J.E. Biester, Vierter Bd., Julius bis December 1784, pp. 175-180.

Ivi, p. 176. 
addirittura carne umana. Secondo Gedike i sacrifici umani erano venuti non alle origini, bensì in un periodo «intermedio di civiltà». Solo il raggiungimento pieno dell'illuminazione (Aufklärung) e della civiltà aveva fatto percepire l'abominio di quelle pratiche ${ }^{6}$. In antitesi all'identità fra essere e mangiare, Gedike ricordava l'affermazione di Cartesio: «cogito ergo sum» ${ }^{7}$. L'autocoscienza dell'uomo civile si fondava sull'identità fra essere e pensiero. Per il teologo dunque al materialismo del primitivo si contrapponeva il razionalismo o lo spiritualismo dell'uomo moderno.

Benché non esistano prove del fatto che Feuerbach abbia letto questo articolo, esso è utile per comprendere il suo distacco da Hegel e i suoi due interventi sull'alimentazione e sui sacrifici. Anch'egli nel suo periodo giovanile aveva sottoscritto la concezione cartesiana, secondo cui l'essenza umana era data dal pensiero o dall'autocoscienza ${ }^{8}$. Poi però nei Principi della filosofia dell'avvenire aveva negato che il carattere distintivo dell'uomo risiedesse semplicemente in quelli. La sua essenza era una totalità che si rifletteva nel complesso della sua sensibilità. Non si trattava solo della capacità di sentire e percepire, bensì di tutta la relazione corporea con la natura. Anche lo stomaco umano era essenzialmente diverso da quello degli animali: «Dagli animali l'uomo non si distingue solo grazie al pensiero. Tutta la sua essenza costituisce piuttosto tale differenza [...] Anzi, persino lo stomaco dell'uomo, malgrado noi lo guardiamo dall'alto in basso con disprezzo non è un'essenza ferina, ma umana, perché è universale, perché non deve servirsi di un tipo determinato di alimenti. Proprio per questo l'uomo non è affetto da quel furore vorace (Freßbegierde) con cui l'animale si getta sulla preda» ${ }^{9}$. L'universalità, che già nell'Essenza del cristianesimo Feuerbach aveva attribuito al genere umano, faceva sì che questo non si limitasse a un tipo di alimenti e quindi dimostrasse, anche nel mangiare, la sua libertà. In proposito egli si appoggiava alla distinzione, presente nella lingua tedesca, fra "essen", mangiare proprio degli umani, e "fressen", divorare tipico degli animali, aggiungendo come nel secondo caso lo stomaco assumesse il predominio su tutto, mentre nel primo caso esso era in armonia con la testa, ossia con la ragione e l'etica. Un amico di famiglia, Georg Friedrich Daumer, criticò l'esaltazione che in questo passo Feuerbach aveva fatto dell'uomo rispetto agli animali. Anzitutto la presunta universalità dello stomaco umano, non circoscritto a un determinato genere di alimenti, non valeva solo per gli uomini, ma anche per alcune specie di animali, come per esempio i maiali che digerivano i più svariati alimenti. Inoltre non si trattava di una caratteristica del tutto positiva. Infatti

\footnotetext{
Ivi, pp. 178-179.

Ivi, p. 177.

Si veda la sua Storia della filosofia moderna, in: Feuerbach, Gesammelte Werke (GW), Hrsg. W. Schuffenhauer, Berlin, Akademie Verlag 1967-2004, II, p. 255: «Il pensiero è quindi la mia sostanza».

9 GW, IX, p. 335, tr. it. Claudio Cesa, qui lievemente modificata, in: Feuerbach, La filosofia dell'avvenire, RomaBari, Laterza 1984, pp. 124-125.
}

\begin{tabular}{|l|l|l|l|l|}
\hline Q Rovista Dialectus & Ano 5 & n. 12 & Janeiro - Julho 2018 & p. 109-120 \\
\hline
\end{tabular}


mentre gli animali più pacifici si limitavano ai vegetali, l'uomo, come i rapaci, si rivolgeva alla carne, talvolta alla carne umana. Così non si poteva dire che non avesse dato prova di voracità. Egli, a differenza di una tranquilla mandria al pascolo, si gettava talvolta sul cibo con avidità e si rimpinzava fino a morirne. In ogni caso bastava vedere un uomo affamato per constatare come perdesse l'autocontrollo ${ }^{10}$. Daumer, pur con evidenti semplificazioni, metteva in rilievo l'ambiguità della presunta universalità sostenuta da Feuerbach. Infatti se riguardava l'ampiezza dei generi alimentari, allora si potevano indicare animali che eguagliavano o addirittura superavano l'uomo. Se invece corrispondeva alla capacità di elevarsi al di sopra del cibo nel suo aspetto materiale, allora doveva essere meglio spiegata. Inoltre Daumer toccava un punto, al quale Feuerbach era stato sensibile fin dai primi scritti, quello della fame.

Nello stesso brano della Storia della filosofia moderna che prima abbiamo ricordato per l'identificazione dell'essenza umana col pensiero, Feuerbach accennava alla fame come esempio per distinguere vari gradi di essere: «Un essere con uno stomaco riempito in modo da star bene è un essere molto più reale di un essere con stomaco vuoto» ${ }^{11}$. Lo stomaco vuoto rappresentava sensibilmente la contraddizione fra la presenza ideale dell'oggetto e la sua assenza reale, ossia la separazione da un ente, al quale per natura si era uniti. Già nella dissertazione De ratione Feuerbach si era soffermato su questa situazione per mettere in luce la tensione dialettica del desiderio ${ }^{12}$. Nelle Lezioni tenute a Erlangen come Privatdozent era tornato sull'argomento trattando dell'impulso o della pulsione (Trieb), un concetto già elaborato da Fichte, che avrebbe avuto grande fortuna nell'Ottocento fino a Freud e al suo famoso saggio della Metapsicologia: Le pulsioni e i loro destini (Triebe und Triebschicksale). Aveva affermato: «Un altro esempio è l'appagamento dell'impulso (Triebes) stesso; quando calmo la fame, questo calmarla in quanto appagamento è affermazione della mia vita, un godimento, ma questo godimento c'è in me soltanto in quanto c'è la fame, il non godimento [...] al godimento spetta l'essere e il non essere» ${ }^{13}$. Nel Leibniz Feuerbach aveva spiegato ulteriormente il rapporto con l'oggetto, presente negli impulsi e, in particolare, nella fame e nella sete: «A dispetto di tutti gli empiristi fame e sete sono due filosofi a priori; esse anticipano e deducono a priori l'esistenza dei loro oggetti» ${ }^{14}$. Nella loro struttura ontica era dunque insito l'oggetto, prima che si presentasse nelle cose sensibili. Questa visione caratterizza nell'Essenza del cristianesimo la relazione del soggetto con l'oggetto: «L'uomo non è nulla senza oggetto [...]

10 Georg Friedrich Daumer, Der Anthropologismus und Kritizismus der Gegenwart in der Reife seiner Selbstoffenbarung, Nürnberg, Bauer \& Raspe 1844, pp. 11-14, 18-20.

11 GW II, p. 252.

12 GW I, pp. 66, 68: «Adest ergo in desiderio id, quod abest».

13 GW XIV, p. 55.

14 GW III, p. 141.

\begin{tabular}{|l|l|l|l|l|}
\hline Q Povista Dialectus & Ano 5 & n. 12 & Janeiro - Julho 2018 & p. 109-120 \\
\hline
\end{tabular}


Tuttavia l'oggetto al quale un soggetto si riferisca essenzialmente, necessariamente non è altro che l'essenza propria di questo soggetto, ma resa oggettiva» ${ }^{15}$. La conclusione dell'opera consisteva nell'esaltazione dell'eucarestia secondo il suo significato etimologico, ossia come solenne ringraziamento per il pane e il vino grazie all'esperienza della fame: «Per comprendere il significato religioso della consumazione del pane e del vino mettiti nella situazione in cui quell'atto altrimenti quotidiano venga, contro natura, violentemente, interrotto. La fame e la sete non distruggono solo la forza fisica, bensì anche quella spirituale e morale dell'uomo, lo spogliano dell'umanità, dell'intelletto, della coscienza. Oh! Se tu mai provassi tale mancanza, tale infelicità, allora come benediresti e loderesti la qualità naturale del pane e del vino che ti ridonano la tua umanità, il tuo intelletto! Così basta solo interrompere il consueto, comune corso delle cose per restituire a quanto è comune un significato non comune, alla vita in quanto tale un significato assolutamente religioso. Santo sia per noi quindi il pane, santo il vino, ma santa anche l'acqua! Amen» ${ }^{16}$.

Nell'imminenza della rivoluzione il tema della fame e dell'alimentazione era tornato. Ne La questione dell 'immortalità dal punto di vista dell'antropologia (1847) si contesta di nuovo la riduzione dell'essenza umana alla testa e si ribadisce che a questa è «unito nel modo più stretto lo stomaco»e che lo stesso «gusto estetico» presuppone il «gusto físico». In quell'occasione Feuerbach aveva anticipato il suo motto: "Ciò che l'uomo è (ist), è indipendente da ciò che mangia (isst)?"17. Si era chiesto: «Ma non è necessario soddisfare la propria fame prima della propria sensibilità artistica? Si possono avere in testa sentimenti estetici e morali quando si ha in corpo fame o sostanze nutritive inadatte allo stomaco umano?». Aveva così indicato nell'alimentazione umana la «prima condizione del modo di pensare e dello sviluppo umani» ${ }^{18}$. Non si trattava solo di mangiare o di non mangiare, ma di scegliere cibi adatti. Fra il tipo di alimentazione e il carattere di un popolo si poteva stabilire una relazione ${ }^{19}$.

Questi spunti ebbero un ampio sviluppo in occasione del libro di Jacob Moleschott, La dottrina degli alimenti. Per il popolo ${ }^{20}$ che Feuerbach recensì verso la fine del 1850, poco dopo il fallimento della rivoluzione in Germania. Feuerbach aveva personalmente seguito i lavori del parlamento di Francoforte dalla parte dei democratici. Quando ormai il loro fallimento era evidente, aveva accettato l'invito degli studenti di Heidelberg a trasferirsi là ad insegnare. Poiché l'università non si era resa disponibile, aveva tenuto le sue lezioni sull'essenza della religione dal 1 dicembre

\footnotetext{
GW V, p. 33, tr. it. Francesco Tomasoni, Roma-Bari, Laterza 1997, p. 28.

Ivi, p. 454, tr. it. p. 288.

GW X, p. 230, tr. it. Marco Vanzulli, Milano, Mimesis 2000, p. 84.

Ivi, p. 260, tr. it., pp. 122-123.

Ivi, p. 230, tr. it. 84-85.

20 Lehre der Nahrungsmittel. Für das Volk, Erlangen, Enke 1850.
}

\begin{tabular}{|l|l|l|l|l|}
\hline Q Revista Dialectus & Ano 5 & n. 12 & Janeiro - Julho 2018 & p. 109-120 \\
\hline
\end{tabular}


1848 al 2 marzo 1849 nel palazzo municipale della città di fronte a studenti, intellettuali e operai ${ }^{21}$. Fra questi c'era Jacob Moleschott (1822-1893), un giovane olandese, iscritto alle scienze naturali, ma interessato anche alla filosofia e, in particolare, all'antropologia. Questi stabilì con Feuerbach un rapporto di amicizia che durò anche dopo che si allontanò dalla Germania. Infatti Moleschott, diventato Privatdozent, incontrò opposizioni sempre più forti nell'università di Heidelberg e nel 1854 dovette emigrare prima in Svizzera, poi in Italia, dove si affermò non solo come uomo di scienza, bensì anche come politico diventando senatore del regno d'Italia.

La recensione di Feuerbach, intitolata Scienza naturale e rivoluzione, colloca il libro di Moleschott in un ampio orizzonte e stabilisce una stretta connessione fra i risultati delle nuove scienze e le rivendicazioni sociali e politiche della rivoluzione. Infatti, come Copernico con le sue teorie astronomiche aveva distrutto il cielo della teologia e mutato la concezione del mondo, così le scoperte riguardanti l'alimentazione erano destinate a cambiare la visione del mondo e le possibilità esistenziali dell'uomo. $\mathrm{E}$ in questo contesto che risuona il motto di Feuerbach: «Se volete migliorare il popolo, dategli, invece di declamazioni contro il peccato, cibi migliori. L'uomo è ciò che mangia. Se mangia solo vitto vegetale, è pure soltanto un ente che vegeta e non ha energia» ${ }^{22}$. Feuerbach riprendeva gli esempi fatti da Moleschott: gli irlandesi, alimentati dal «fiacco sangue di patate», avevano ben poche prospettive di successo sugli inglesi, nutriti dalle loro mandrie ${ }^{23}$. Proprio fra il 1845 e il 1848 l'Irlanda aveva subito una pesante carestia per l'invasione della peronospora che aveva distrutto gran parte dei raccolti. La popolazione era calata del 20\%: un milione di persone erano morte e circa 2 milioni erano emigrate negli Stati Uniti. L'osservazione di Moleschott, ripresa da Feuerbach, aveva dunque una forte attualità. Altri esempi riguardanti un'alimentazione carente, quasi esclusivamente vegetariana, riguardavano gli italiani, gli indù e gli abitanti dei tropici. Feuerbach però applicava il discorso anche ai tedeschi e al fallimento della loro rivoluzione: «Da qui si spiega anche fra di noi la vittoria della reazione, l'andamento e l'esito vergognoso della nostra cosiddetta rivoluzione di marzo, infatti anche fra di noi la stragrande maggioranza del popolo sussiste solo rimpinzandosi con e attraverso patate ${ }^{24}$. Con questo discorso Feuerbach, sulla scorta di Moleschott, non respingeva in assoluto la dieta vegetariana, ma richiedeva che contenesse proteine e quindi includesse le leguminacee: «Dobbiamo per questo

21 Cfr. Werner Schuffenhauer, Ludwig Feuerbach im Revolutionsjahr, in: Philosophie - Wissenschaft - Politik, Berlin, Akademie Verlag 1982, pp. 189-205, spec. pp. 195-200; Erich Thies, Ludwig Feuerbach zwischen Universität und Rathaus oder die Heidelberger Philosophen und die 48er Revolution, Heidelberg, Verlag Brigitte Guderjahn 1990, pp. 56-85.

22 GW X, p. 367, tr. it. Francesco Tomasoni, in: Feuerbach, L'uomo è ciò che mangia, Brescia, Morcelliana 2015, p. 50 .

23 Ibid.

24 GW X, 367, tr. it., p. 51.

\begin{tabular}{|l|l|l|l|l|}
\hline Qenista Qialectus & Ano 5 & n. 12 & Janeiro - Julho 2018 & p. 109-120 \\
\hline
\end{tabular}


disperare? C'è una sostanza che può sostituire, anche presso la classe più povera, la patata e nello stesso tempo è in grado di infondere nel popolo energia e sentimenti virili? Sì, esiste una tale sostanza, una sostanza quindi che è la garanzia di un futuro migliore, che contiene il germe di una nuova rivoluzione, lenta e graduale, ma altrettanto più solida: è la legumina» ${ }^{25}$. Essenziale era che il cibo contenesse gli elementi di cui è composto l'uomo. La loro identità dinamica era costituita dal sangue: «I cibi diventano sangue, il sangue cervello e cuore, sostanza del pensiero e dei sentimenti ${ }^{26}$. Fin dalla prima pagina dell'Introduzione Moleschott aveva descritto nel suo libro questo movimento ascendente dagli alimenti al cervello chiedendosi se i primi non condizionassero «l'ardore del cuore, la forza del muscolo, la solidità delle ossa e la vivacità del cervello» ${ }^{27}$. Per contrasto egli aveva subito accennato alle conseguenze nefaste della fame che appunto dimostrava come lo spirito non fosse «indipendente dalla materia». ${ }^{28}$ Nel seguito egli aveva spiegato le alterazioni subite dall'affamato non solo nella respirazione e nel battito cardiaco, ma anche nelle sensazioni del gusto, della vista e dell'udito, come pure in uno stato psichico sempre più inquieto e nell'oscuramento del giudizio. Finiva così per gettarsi sui cadaveri e sulle carogne ${ }^{29}$. Feuerbach, colpito da questa descrizione «delle terribili conseguenze della fame», commentò il fatto che il «nulla nello stomaco» fosse «molto reale», a differenza del «nulla nella testa» ${ }^{30}$. Con quest'ultima espressione Feuerbach alludeva alla questione speculativa del non essere. Come sappiamo, nel periodo giovanile egli aveva spiegato la contraddizione fra essere e non essere anche attraverso l'esempio della fame. Ora però questa diventa la prova lampante della differenza fra i due termini, una differenza sperimentabile in modo drammatico. Infatti spiega Feuerbach: «Quali spinose analisi ha procurato ai filosofi l'essere! È uno o molteplice, fa tutt'uno con il pensatore o è diverso dal nulla del pensiero? Inutili questioni! L'essere fa tutt'uno con il mangiare; essere significa mangiare; ciò che è (ist) mangia (isst) e viene mangiato. Mangiare è la forma soggettiva, attiva, essermangiato la forma oggettiva, passiva dell'essere, ma entrambe sono inseparabili. Soltanto nel mangiare perciò si riempie il vuoto concetto di essere e diventa palese l'assurdità della questione se l'essere e il non-essere, ossia se il mangiare e la fame si identifichino» ${ }^{31}$.

Nel suo aspetto biologico la fame era dovuta all'impoverirsi del sangue che non poteva più rifornire i vari tessuti organici. A questo proposito Moleschott aveva descritto la grande circolazione che dal cuore per mezzo delle arterie andava agli «organi più lontani» e poi, dopo aver

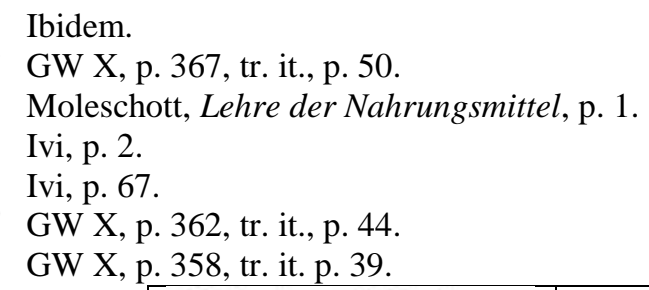

\begin{tabular}{|l|l|l|l|l|}
\hline Rovista Dialectus & Ano 5 & n. 12 & Janeiro - Julho 2018 & p. 109-120 \\
\hline
\end{tabular}


effettuato scambi con questi, ritornava attraverso le vene ${ }^{32}$. Già Feuerbach aveva ammirato questo dinamismo e nell'Essenza del cristianesimo l'aveva paragonato alla trasposizione dell'essenza umana in Dio e al suo recupero: «Come la circolazione arteriosa spinge il sangue fino alle più lontane estremità, mentre quella venosa lo riporta indietro, come la vita in genere consiste in una perenne sistole e diastole, così è anche la religione. Nella sistole religiosa l'uomo espelle lontano da sé la sua propria essenza, respinge, rigetta se stesso; nella diastole religiosa riprende nel suo cuore l'essenza espulsa» ${ }^{33}$. La circolazione del sangue era simbolo, per Feuerbach, del rapporto religioso, anzi della vita in genere, per Moleschott addirittura dell'intero moto cosmico, costituito da «una circolazione eternamente incessante» ${ }^{34}$. La natura superava le barriere fra le varie classi, sicché il naturalista poteva andare oltre le distinzioni limitanti l'intelligenza umana ${ }^{35}$. La stessa opposizione fra morte e vita veniva meno, giacché la prima era riassorbita nel ciclo incessante di trasformazione. La decomposizione di alcune sostanze era un passaggio verso la formazione di altre. «Ciò che durante la vita e dopo la morte è incessantemente ceduto da uomini, animali e piante in decomposizioni molteplicemente concatenate fra loro ritorna nell'aria e nel suolo per formare di nuovo gli alimenti delle piante» ${ }^{36}$. A questo argomento Moleschott avrebbe dedicato ben presto il libro intitolato La circolazione della vita (1852) e avrebbe esaltato nella natura la «forza creatrice e organizzatrice che fa apparire le piante come il regno fiorito dell'inconscia poesia». Questo afflato di romantico naturalismo l'avrebbe portato a esaltare la vittoria della vita sulla morte: «C'è morte nella vita e vita nella morte. Questa morte non è una morte tetra, spaventosa. Infatti nell'aria e nella putredine si librano e si posano i germi eternamente turgidi dei fiori. Chi conosce la morte in questa connessione, ha compreso l'inesauribile energia motrice della vita e con essa l'intera pienezza della poesia umana che poggia immutabilmente sulle colonne marmoree della verità» ${ }^{37}$. Nel superamento ininterrotto della morte da parte della vita la natura avrebbe attuato la sua immortalità e quindi il trionfo del bene. Da qui l'affermazione: «Perciò il bene non può perire» ${ }^{38}$. Ben diversi erano stati gli accenti di Schopenhauer sulla circolazione della materia che per lui sanciva l'ineluttabile distruzione dell'individuo da parte degli elementi chimici, dai quali esso dipendeva ${ }^{39}$.

32 Moleschott, Lehre der Nahrungsmittel, p. 53.

${ }^{3}$ GW V, p. 73, tr. it. p. 48.

${ }^{34}$ Moleschott, Lehre der Nahrungsmittel, p. 49.

35 Ivi, p. 50.

36 Ivi, p. 48.

37 Moleschott, Der Kreislauf des Lebens. Physiologische Antworten auf Liebig's Chemische Briefe, Mainz, Zabern 1852, p. 438, tr. it. Francesco Tomasoni, in: Materialisti dell'Ottocento, a cura di Arrigo Pacchi, Bologna, Il Mulino 1978, p. 173.

38 Ivi, p. 434, tr. it., p. 171.

39 Arthur Schopenhauer, Parerga und Paralipomena II, Zürich, Haffmanns Verlag 1988, p. 260.

\begin{tabular}{|l|l|l|l|l|}
\hline Revista Qialectus & Ano 5 & n. 12 & Janeiro - Julho 2018 & p. 109-120 \\
\hline
\end{tabular}


Feuerbach nella sua recensione ha rivolto l'attenzione al tema della circolazione vitale, toccato da Moleschott anche nel suo primo libro, e ha ripreso in senso politico e sociale l'esaltazione della natura come capace di superare le barriere politiche ${ }^{40}$. In questa prospettiva la fame che attanagliava larghi strati della popolazione era un fenomeno innaturale, come dimostravano gli effetti dell'ossigeno. Moleschott ne aveva parlato a proposito della piccola circolazione dal cuore ai polmoni spiegando come l'ossigeno rendesse possibile l'alimentazione dei tessuti attraverso il sangue arterioso, ma provocasse anche la combustione e la corruzione degli elementi ${ }^{41}$. Feuerbach ne riprendeva il discorso ricordando che quell'elemento di vita si trasformava in potere di morte ${ }^{42}$, ma vedeva l'attuarsi di ciò soprattutto per le diseguaglianze sociali che condannavano alla fame. Anche dalla spiegazione della digestione e dell'assimilazione, fornita da Moleschott, Feuerbach ricavava una connotazione positiva rispetto alla natura. Il giovane scienziato olandese aveva descritto il lungo processo di sminuzzamento e di scomposizione delle sostanze, come pure l'escrezione per opera dei polmoni, dei reni e dell'intestino ${ }^{43}$. Aveva mostrato come l'uguaglianza fra la natura esteriore e il corpo umano fosse un risultato raggiunto solo mediante sacrifici e distruzione. Feuerbach vi si allacciava richiamando la necessaria «serie di mediazioni e di trasformazioni», ma si soffermava soprattutto sul significato ontologico dell'esito finale: «in noi non c'è nulla che non sia anche fuori di noi» ${ }^{44}$. A questo proposito egli poteva applicare all'alimentazione quel rapporto di identità fra soggetto e oggetto che aveva visto in Leibniz e in Hegel e che aveva utilizzato nell'Essenza del cristianesimo $^{45}$ : gli oggetti che assimiliamo sono quelli che costituiscono noi stessi, il nostro sangue. Da qui il senso del motto: 1'uomo è ciò che mangia. Mentre attua la propria esistenza, entra in comunione col tutto: «l'alimentazione è 'l'uno e tutto" di Spinoza che tutto comprende in sé, l'essenza delle essenze» ${ }^{46}$.

In questo orizzonte si comprendono le affermazioni: «La base della cultura e dei sentimenti umani è una alimentazione umana» e «La dieta è la base della sapienza e della virtù» ${ }^{47}$. Esse non possono essere prese come espressione di un materialismo deterministico, secondo cui un cibo più raffinato produrrebbe pensieri, sentimenti o comportamenti migliori. Fra le condizioni materiali e l'attività culturale Feuerbach ha sempre stabilito una differenza che salvaguardasse 1'autonomia dei due ambiti. Quando nel Prologo ai Sämmtliche Werke si è interrogato sul senso

\footnotetext{
${ }^{40}$ Feuerbach, GW X, pp. 349-350, 357, 366, tr. it., pp. 28-29, 37, 49.

${ }^{41}$ Moleschott, Lehre der Nahrungsmittel, pp. 49, 52.

42 Feuerbach, GW X, p. 361, tr. it. p. 43, cfr. Moleschott, Lehre der Nahrungsmittel, p. 49.

43 Moleschott, Lehre der Nahrungsmittel, pp. 20, 59.

44 GW X, pp. 357, 365, pp. 37, 48.

45 Si veda il passo sopra citato, GW V, pp. 33-34, tr. it., pp. 28-29.

46 GW X, p. 358 , tr. it. p. 38.

47 GW X, pp. 367, 368, tr. it., pp. 50, 52.
}

\begin{tabular}{|c|c|c|c|c|}
\hline Rovista Dialectus & Ano 5 & n. 12 & Janeiro - Julho 2018 & p. $109-120$ \\
\hline
\end{tabular}


della sua attività intellettuale egli ha sì ammesso l'importanza di affrontare $\mathrm{i}$ «mali dello stomaco», ma ha aggiunto che molti di questi avevano il loro fondamento nella testa ${ }^{48}$. Quando, verso la fine della vita, fu indotto a precisare il suo pensiero rispetto al materialismo di Vogt, Moleschott e Ludwig Büchner egli rispose: «L'uomo è per me come per loro un ente naturale, sgorgato dalla natura, ma mio oggetto principale sono i pensieri e gli enti fantastici che, sorti dall'uomo, valgono per enti reali nell'opinione e nella tradizione degli uomini» ${ }^{49}$. Che significa allora indicare l'alimentazione come base della sapienza? Anzitutto che l'uomo non esiste senza la natura, che la sua vita non è possibile senza materia, che il suo pensiero non c'è senza attività del cervello e quindi senza fosforo, un motto ripreso da Moleschott stesso ${ }^{50}$. Più profondamente però l'alimentazione ci mostra l'esempio di una natura comunitaria: «Ora sappiamo che la respirazione è la fonte principale del calore, che l'aria è una parte essenziale di noi stessi, che noi pompiamo tutto dall'esterno, che non abbiamo nulla di proprio, che veniamo al mondo come perfetti pitocchi e comunisti, che in noi non c'è nulla che non sia anche fuori di noi, che in fondo noi siamo rappezzati soltanto con ossigeno, azoto, carbonio e idrogeno, questi pochi semplici elementi» ${ }^{51}$.

Il motivo della comunione è dominante anche nel secondo saggio, caratterizzato dalle indagini filologiche sui poemi omerici, sulle religioni dei primitivi e sulla Bibbia che Feuerbach aveva condotto nella Teogonia (1857). Esso, intitolato Il segreto del sacrificio ovvero l'uomo è ciò che mangia, fu pubblicato nel 1866 all'interno del decimo volume delle Opere, curate da Feuerbach stesso, ma dovrebbe essere stato scritto nel $1862^{52}$. Rivela un cambiamento di prospettiva nella valutazione del sacrificio. Nell'Essenza del cristianesimo il sacrificio era stato visto come l'espressione dell'estraniazione religiosa che portava a negare l'umano. Emblematici erano i «sacrifici umani cruenti» ${ }^{53}$. Nell'Essenza della religione tale aspetto drammatico era stato ricondotto al senso di colpa provato dall'uomo nel consumare i frutti della natura ${ }^{54}$. In questo saggio predomina l'aspetto positivo: la comunione. Perfino l'olocausto che attraverso il fuoco bruciava tutta la vittima e rappresentava il dono totale alla divinità ${ }^{55}$, è inteso come un atto supremo di comunione: «"Venne dal Signore il fuoco e consumò, letteralmente: mangiò sull'altare l'olocausto e

48 GW X, pp. 189-190.

49 Lettera a Gustav Bäuerle, 31 maggio 1867, GW XXI, pp. 302-303. Per il dibattito sul materialismo di Feuerbach mi permetto di rinviare a Tomasoni, Ludwig Feuerbach. Biografia intellettuale, Brescia, Morcelliana 2011, pp. 321326, 364, 381, 383, 384, 385-387, 389-391, 412, 437-440.

50 GW X, p. 360, tr. it., p. 41, cfr. Moleschott, Lehre der Nahrungsmittel, p. 116,

51 GW X, p. 357, tr. it., p. 37.

52 Cfr. sua lettera a Wilhelm Bolin del 5 novembre 1862, GW XXI, p. 41.

53 GW V, p. 446, tr. it., p. 283.

54 GW X, p. 33.

5 Cfr. Gerhard Von Rad, Theologie des Alten Testaments, I, München, Kaiser Verlag 1968, p. 268.

\begin{tabular}{|l|l|l|l|l|}
\hline Q Revista Dialectus & Ano 5 & n. 12 & Janeiro - Julho 2018 & p. 109-120 \\
\hline
\end{tabular}


il grasso"; quindi questo consumare o mangiare è in intima relazione con le oblazioni» ${ }^{56}$. Karoly Kerényi ha mostrato la presenza nei sacrifici di due componenti: la distruzione e la comunione. La prima sarebbe evidente nel rituale di sgozzamento delle vittime, accuratamente descritto dai poemi antichi, la seconda nel banchetto sacrificale, nel quale si immaginano gli dei come commensali degli uomini ${ }^{57}$. Feuerbach ha accentuato il lato positivo attraverso l'enfasi sul mangiare che confermerebbe l'identità essenziale fra la divinità e l'uomo, pur con la differenza immaginata fra l'essenza ideale e quella reale: "Gli dei sono ciò che è l'uomo, perciò mangiano ciò che mangia l'uomo, "siedono al nostro banchetto e mangiano con noi, come noialtri". Stessa essenza, stessi cibi e viceversa. Il bisogno di cibo è proprio dell'uomo per se stesso, ma il godimento di esso è condiviso con gli dei [...] L'onore del godimento di cibi e bevande spetta e appartiene agli dei, ma la fatica connessa [...] il bisogno e lo scorno restano in dote agli uomini» ${ }^{58}$.

Nel banchetto sacrificale l'uomo, mangiando, esprimeva la sua essenza divina. Da qui la correzione che Feuerbach apporta alla interpretazione che nell' Essenza del cristianesimo aveva dato all'episodio del banchetto dei settanta anziani sul monte alla presenza di Dio (Es. 24,10-11). Là era stato considerato come la prova del fatto che gli ebrei avessero sviluppato solo i sensi «gastrici»: «La vista della suprema essenza stimolò dunque in loro solo l'appetito a mangiare» ${ }^{59}$. Ora quel banchetto diventa manifestazione di una gioia profonda: «Si rallegrarono della vita, infatti mangiare e vivere sono un tutt'uno» ${ }^{60}$.

Come abbiamo visto nel primo saggio, non ogni cosa è immediatamente disponibile come cibo umano. Generalmente occorre un processo di trasformazione, come pure una scelta da parte dell'uomo: «Noi mangiamo e digeriamo di un animale o di una pianta appunto solo ciò che è uguale a noi, alla nostra essenza, ciò che può essere indirettamente carne e sangue umano» ${ }^{61}$. Ora però nella scelta degli alimenti Feuerbach sottolinea il significato culturale, mediante il quale un popolo esprime la sua identità differenziandosi dagli altri: «Com'è il cibo, così è l'essenza, com'è l'essenza, così è il cibo. Ciascuno mangia appunto ciò che è conforme alla sua individualità $\mathrm{o}$ natura, alla sua età, al suo genere, al suo ceto o professione, al suo grado. Questo "non è mangiare per uomini, ma solo per scrofe", "non è mangiare per signori, ma solo per bifolchi”. Siete "mangia asini o mangia gatti” _ questi insulti, che un tempo si lanciavano gli abitanti di città vicine in Germania, suonavano così offensivi da indurre a scontri sanguinosi» ${ }^{62}$. La scelta del cibo assume

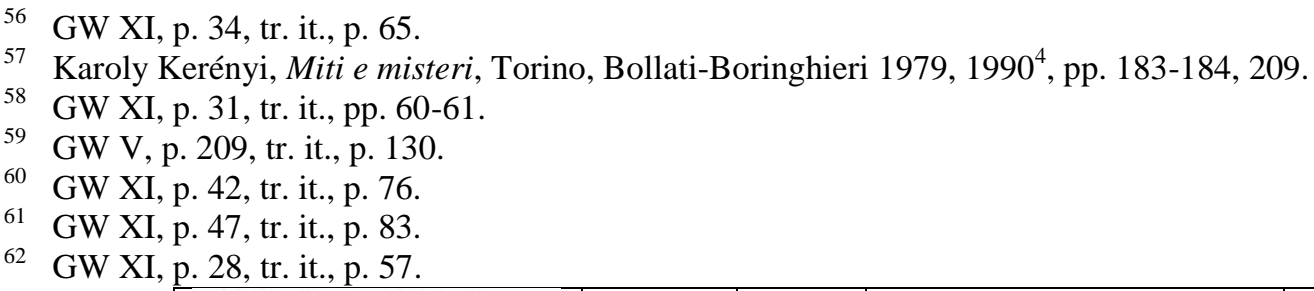

\begin{tabular}{|l|l|l|l|l|}
\hline Q Povista Dialectus & Ano 5 & n. 12 & Janeiro - Julho 2018 & p. 109-120 \\
\hline
\end{tabular}


perfino un significato religioso, come si vede nelle prescrizioni alimentari ebraiche, motivate dalla santità di Dio: «Infatti io sono il Signore, il vostro Dio. Dovete santificarvi in modo da essere santi, infatti io sono santo, e non dovete macchiare la vostra anima con qualsiasi animale strisciante che serpeggia sulla terra» ${ }^{63}$. La spiegazione di Feuerbach è la seguente: «L'uomo è ciò che mangia; chi mangia qualcosa di abominevole, è lui stesso abominio» ${ }^{64}$. Il cibo dunque è portatore di significati culturali e religiosi che costituiscono l'identità dell'uomo, anzi di un popolo e di un individuo.

In questa prospettiva si richiamano alcuni elementi della dieta umana. Anzitutto il grano, la farina e il pane, simboli di fecondità, ma anche di caducità e morte, a tal punto che gli uomini sono «i mortali che mangiano il frutto della terra» ${ }^{65}$. Un ingrediente essenziale è il sale «simbolo di amicizia, di fedeltà», ma anche di arguzia, a tal punto che Feuerbach riprende il motto di Moleschott riguardo al fosforo e lo rielabora nel modo seguente: «senza sale niente arguzia, niente sagacia» ${ }^{66}$. Anche il sangue, costante nei sacrifici, è riconosciuto come parte dell'alimentazione e come simbolo di vita ${ }^{67}$. Ad esso viene accostato il vino che «allieta allo stesso modo dei e uomini» ${ }^{68}$. Il significato culturale, che in questo saggio è attribuito all'alimentazione, spiega la differenza fra il mangiare animale e quello umano, di cui aveva parlato nei Principi e che Daumer aveva criticato per l'oscurità e ambiguità delle espressioni. L'alimentazione nella sua globalità arriva a comprendere non solo il cibo propriamente detto, ma anche l'aria: «Appunto l'uomo è ciò che mangia. Egli però mangia non solo mediante l'esofago; egli mangia anche per mezzo della trachea che, anzi, si antepone. Mangiare o bere aria significa respirare. Gli antichi chiamavano perciò l'aria un cibo, un nutrimento e, in verità, in modo assolutamente corretto, infatti solo per la cooperazione dell'aria, per l'immissione dell'ossigeno il cibo diventa sangue arterioso. Chi però non sa quanto la condizione dell'essere e dell'essenza umana dipenda dalla condizione e dalla mescolanza di questo cibo etereo? Chi può negare che «puro essere» e «puro pensare» avvengano solo nell'aria pura e che in generale quando si cessa di godere dell'aria, termini anche la vita, anche il pensiero?» ${ }^{69}$. Nel suo sforzo di comprensione globale Feuerbach giunge qui a dire che si «mangia anche con i sensi», secondo l'espressione «mangiare con gli occhi» e che quanto è fatto proprio dai sensi viene poi digerito dal cervello che può essere inteso come «lo stomaco, l'organo di digestione dei sensi». In questa visione si può certamente cogliere l'aspetto materialistico, in base al quale esiste una indissolubile connessione fra corpo e pensiero. Tuttavia si può anche capovolgere

\footnotetext{
GW XI, p. 43, tr. it., p. 77.

GW XI, p. 43, tr. it., p. 78.

65 GW XI, pp. 27-28, tr. it., p. 56.

66 GW XI, pp. 37, 46, tr. it. pp. $69,81$.

67 GW XI, pp. 41-42, tr. it., p. 75.

${ }^{68}$ GW XI, p. 41, tr. it., pp. 74-75.

69 GW XI, p. 44 , tr. it. p. 78.
}

\begin{tabular}{|l|l|l|l|l|}
\hline Q Revista Dialectus & Ano 5 & n. 12 & Janeiro - Julho 2018 & p. 109-120 \\
\hline
\end{tabular}


tale conseguenza e vedere come «il cibo non abbia solo significato corporeo, bensì anche spirituale», come Feuerbach sottolinea ${ }^{70}$. Anche qui Feuerbach è riuscito a mostrare la profondità di un argomento che i filosofi avevano comunemente accantonato come banale e che invece era importante per chi già nel 1839, nell'ultima pagina di Per la critica della filosofia hegeliana aveva invocato $\ll$ il ritorno alla natura» come $\ll$ unica forma di salvezza» ${ }^{71}$.

71 GW IX, p. 61, tr. it. Claudio Cesa in: Feuerbach, La filosofia dell'avvenire, p. 52.

\begin{tabular}{|l|l|l|l|l|}
\hline Qenista Qialectus & Ano 5 & n. 12 & Janeiro - Julho 2018 & p. 109-120 \\
\hline
\end{tabular}

